\title{
Fractional Brownian motion in crowded fluids $\dagger$
}

\author{
Dominique Ernst, $\ddagger^{a}$ Marcel Hellmann, $\ddagger^{b}$ Jürgen Köhler ${ }^{* a}$ and Matthias Weiss ${ }^{* b}$ \\ Received 30th January 2012, Accepted 22nd March 2012 \\ DOI: $10.1039 / \mathrm{c} 2 \mathrm{sm} 25220 \mathrm{a}$
}

\begin{abstract}
Diffusion in crowded fluids, e.g. in the cytoplasm of living cells, has frequently been reported to show anomalous characteristics (socalled 'subdiffusion'). Several random walk models have been proposed to explain these observations, yet so far an experimentally supported decision in favor of one of these models has been lacking. Here, we show that experimentally obtained trajectories in a prototypical crowded fluid show an asphericity that is most consistent with the predictions of fractional Brownian motion, i.e. an anticorrelated, anti-persistent generalization of normal Brownian motion that is related to the fluid's viscoelasticity.
\end{abstract}

Macromolecular crowding, i.e. a total concentration of a variety of macromolecules up to $400 \mathrm{mg} \mathrm{ml}^{-1}$, is a common phenomenon in intracellular fluids. ${ }^{1}$ Crowding can have a considerable impact on (bio)chemical reactions, ${ }^{2}$ hence challenging insights derived from biochemical assays in dilute aqueous solutions. The phosphorylation pattern of the mitogen-activated protein kinase (MAPK), for example, has been shown to vary greatly with the degree of cytoplasmic crowding: ${ }^{3}$ In dilute solutions, MAPK was twice phosphorylated by its kinase in a distributive manner, whereas adding artificial crowding agents resulted in a processive phosphorylation and hence a more efficient activation of MAPK. Recently, a theoretical explanation of these results has been given in terms of crowding-induced anomalous diffusion. ${ }^{4}$ Indeed, crowding is known to strongly alter the diffusional mobility of macromolecules. ${ }^{5}$ Apart from a mere reduction of the diffusion coefficient, i.e. an increased viscosity of the fluid, anomalous diffusion has also been frequently observed in crowded fluids in vivo ${ }^{6-10}$ and in vitro. ${ }^{11-15}$ Here, the mean square displacement (MSD) of a diffusing particle was shown to scale over several decades as $\left\langle r(t)^{2}\right\rangle \sim t^{\alpha}$ with $\alpha<1$ ('subdiffusion').

In spite of the frequent observation of subdiffusion, even in fairly unstructured fluids in vitro, an experimentally supported and unambiguous explanation of the effect in terms of a random walk model has remained elusive. So far, three types of random walks have been considered as an explanation of crowding-induced subdiffusion: (1) Obstructed diffusion (OD), i.e. the motion of a tracer particle in

\footnotetext{
${ }^{a}$ Experimental Physics IV, University of Bayreuth, 95440 Bayreuth, Germany. E-mail: juergen.koehler@uni-bayreuth.de

${ }^{b}$ Experimental Physics I, University of Bayreuth, 95440 Bayreuth, Germany. E-mail: matthias.weiss@uni-bayreuth.de

$\uparrow$ Electronic supplementary information (ESI) available: Experimental and Numerical Methods. See DOI: 10.1039/c2sm25220a

\$ These authors contributed equally to this work.
}

a maze of immobile obstacles, ${ }^{16}$ (2) fractional Brownian motion (FBM) due to the viscoelasticity of the crowded fluid, ${ }^{15}$ and (3) a continuous time random walk (CTRW) in which the diffusing tracer takes power-law distributed rests between periods of free diffusion. The CTRW model is special since it shows weak ergodicity breaking ${ }^{17,18}$ whereas OD and FBM are ergodic random processes with stationary increments. Recent experimental data have indicated that CTRW may be less well suited to explain crowding-induced subdiffusion $^{15,19}$ at least on short and intermediate time scales. ${ }^{20}$

The main problem in relating experimental data to the above models is a lack of detailed information on the diffusion process: several techniques, e.g. fluorescence correlation spectroscopy, only report the MSD and leave all higher moments of the diffusion propagator undetermined. Single-particle tracking (SPT) techniques allow one to record individual trajectories and hence can overcome this limitation. ${ }^{21,22}$ However, precise position determination in SPT requires the collection of many photons of the moving tracer which sets limitations to the temporal resolution and the overall length of the recorded trajectory (due to bleaching of the dye). Yet, an unambiguous deciphering of the random walk model from fairly short SPT trajectories, often accompanied by an unfavorable spatial and temporal resolution, is challenging.

Here, we have utilized a fast and precise single-particle tracking technique to record particle trajectories with a length of $10^{5}$ positions and a spatio-temporal resolution of $10 \mathrm{~nm}$ and $4 \mathrm{~ms}$. From trajectories in prototypical crowded and purely viscous fluids, we have determined the time- and ensemble-averaged MSD of the diffusing particle as well as the random walk's asphericity. As a result, we have found that a transient, yet long-lasting subdiffusion emerged in a crowded but not in a purely viscous fluid. The anomaly was associated with an ergodic mode of motion as evidenced by a recently introduced ergodicty breaking parameter. Comparing the random walks' asphericity with those predicted by computer simulations of normal Brownian motion, FBM, CTRW, and OD, we have found that our experimental data in crowded fluids are best described by the FBM model. Since FBM is closely related to viscoelasticity, we put forward the hypothesis that macromolecular crowding equips fluids with viscoelastic properties that enforce a fractional Brownian motion of diffusing tracer particles.

Single-particle tracking (SPT) is frequently limited by a poor temporal and/or spatial resolution as well as fairly short trajectories. These limitations can be overcome using a tracking technique that has been developed within the last few years: ${ }^{23-26} \mathrm{~A}$ Gaussian focus circles at high speed around a fluorescent particle with the particle 
located at the position of the steepest gradient of the excitation intensity. Taking a diffusion step to escape this position is compensated by moving the sample stage via a negative feedback loop. Hence, the two-dimensional center-of-mass motion can be tracked with a high spatial and temporal resolution.

Using this approach (see the ESI $\dagger$ for the schematic setup and technical details), we were able to track fluorescent beads (diameter $50 \mathrm{~nm}$ ) for up to ten minutes with a temporal resolution of $\Delta t=4 \mathrm{~ms}$ and a spatial accuracy of $\Delta r=10 \mathrm{~nm}$. We have tracked particles in two prototypical fluids: (i) in a purely viscous solution obtained by mixing $60 \%$ sucrose (per weight) into water, and (ii) in a crowded fluid, where $30 \%$ dextran $(500 \mathrm{kDa})$ was dissolved in water. For the latter, anomalous diffusion has already been reported previously. ${ }^{6,12}$ From a slightly higher number of acquired trajectories, we have retained for each fluid only those 21 SPT trajectories for further analysis that contained $4.5 \times 10^{4}-1.5 \times 10^{5}$ positions without blanks, i.e. we discarded those few trajectories in which a weak emission signal lead to a transient loss of the tracked bead. The chosen trajectories did not show any signs of drift. Representative trajectories for both fluids are shown in the ESI.†

As a first step in the analysis, we calculated for each of the selected time traces $r_{i} \equiv r(t=i \Delta t)$ the time-averaged MSD,

$$
\left\langle r(t)^{2}\right\rangle_{T}=\frac{1}{N-k} \sum_{i=1}^{N-k}\left(r_{i}-r_{i+k}\right)^{2} .
$$

Representative time-averaged MSDs for sucrose and dextran solutions are shown in Fig. 1a. To highlight the emergence of a diffusion anomaly, we have divided out the leading order of the MSD, i.e. we have plotted $D(t)=\left\langle r(t)^{2}\right\rangle_{T} I t$ as a function of $t$. While the purely viscous sucrose solution yielded a horizontal line, $D(t)=$ const., a transient power-law decay emerged for the crowded fluid. From the transient scaling $D(t) \sim 1 / t^{0.2}$ (obtained within the grey shaded region), we inferred $\left\langle r(t)^{2}\right\rangle_{T} \sim t^{0.8}$ for small and intermediate time scales. This observation is in quantitative agreement with previous reports ${ }^{15}$ on similar probes. Beyond $t \approx 1 \mathrm{~s}$ a crossover towards normal diffusion emerges, i.e. $D(t)$ tends towards a horizontal line. Indeed, this behavior is expected for all of the above mentioned random walk models for subdiffusion since adapting them to a physical sample requires specification of a minimum and maximum length/time scale.

To determine the anomaly for each trajectory, we restricted the fitting process to the temporal range $50 \mathrm{~ms} \leq t \leq 500 \mathrm{~ms}$ which is not affected by some remaining inertia traces of the setup $(t<50 \mathrm{~ms}$; see discussion in the ESI $\dagger$ ) but also does not suffer from the emerging crossover to normal diffusion at large time scales. The resulting anomaly values, $\alpha$, for all trajectories are summarized in Fig. 1b. A clear separation of the data for the purely viscous sucrose solution (average: $\langle\alpha\rangle=0.98$ ) and the results for a crowded dextran solution (average: $\langle\alpha\rangle=0.82$ ) can be seen.

From the observation $\left\langle r(t)^{2}\right\rangle_{T} \sim t^{\alpha}$ we can already infer that the CTRW model with its distinct weak ergodicity breaking cannot describe the experimental data since it predicts ${ }^{17,18}\left\langle r(t)^{2}\right\rangle_{T} \sim t$. Indeed, even for a truncated CTRW model with only a transient scaling $p(\tau)$ $\sim \tau^{-(1+\alpha)}$ of the distribution of waiting times one observes $\left\langle r(t)^{2}\right\rangle_{T} \sim t$ (cf. ESI $\dagger$ ). Hence, even a more realistic adaptation of the CTRW model appears incompatible with our experimental data.

Next, we calculated for all trajectories an ergodicity parameter ${ }^{17}$ that vanishes if ergodicity is preserved:
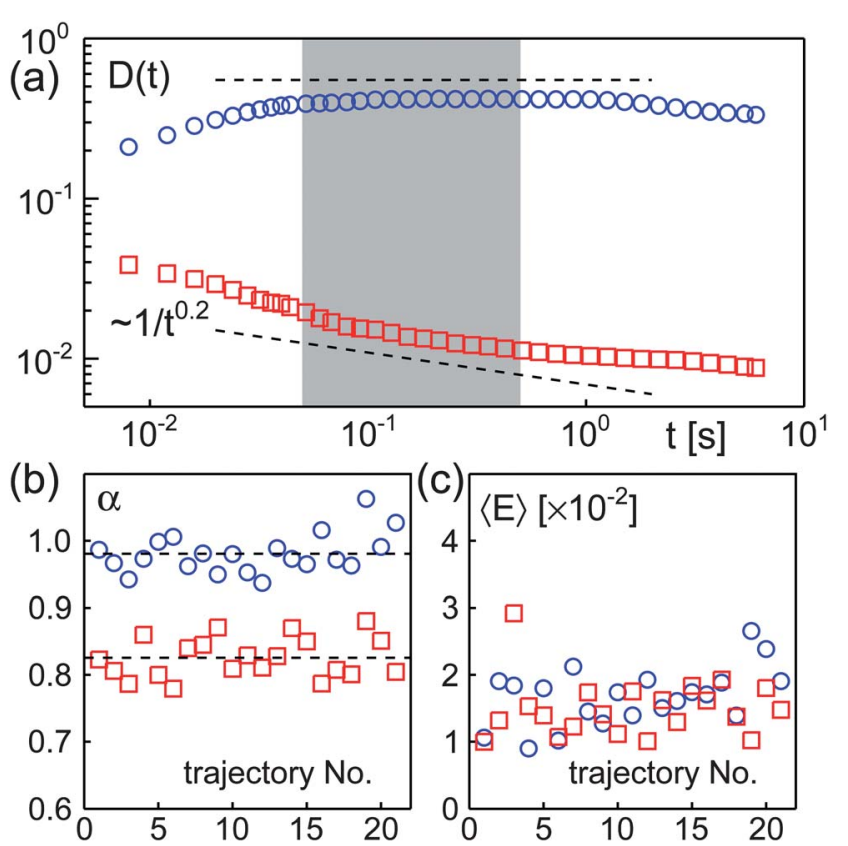

Fig. 1 (a) Representative time-averaged MSD, shown as $D(t)=\left\langle r(t)^{2}\right\rangle_{T} / t$ to highlight the asymptotic scaling. Data for sucrose solutions (blue circles) follows the anticipated scaling for normal diffusion $(D(t)=$ const.). In contrast, data for dextran solutions (red squares) shows a transient subdiffusion (dashed line, $D(t) \sim 1 / t^{0.2}$ ). For $t>1 \mathrm{~s}$ a crossover to the asymptotic scaling $(\alpha=1, D(t)=$ const.) is visible. The grey shaded region indicates the temporal window in which the curves were fitted to extract the anomaly $\alpha$. (b) Anomaly values $\alpha$ for each trajectory as obtained from fitting the time-averaged MSD in the indicated time window. A clear separation between a sucrose solution (blue circles, $\langle\alpha\rangle=0.98$ ) and a crowded dextran solution (red squares, $\langle\alpha\rangle=0.82$ ) is evident. (c) The ergodicity breaking parameter $\langle E\rangle$ [eqn (2)] of all trajectories was very small, indicating ergodicity.

$$
E(t)=\frac{\left\langle\left\langle r(t)^{2}\right\rangle_{T}^{2}\right\rangle_{E}-\left\langle\left\langle r(t)^{2}\right\rangle_{T}\right\rangle_{E}^{2}}{\left\langle\left\langle r(t)^{2}\right\rangle_{T}\right\rangle_{E}^{2}}
$$

To this end, we have cut each trajectory into segments of $N=3000$ time steps and used these segments for the ensemble averaging $\langle\ldots\rangle_{E}$. As a result, we observed that for all trajectories $\langle E\rangle \leq 0.03$ (Fig. 1c). Here, the average of $E(t)$ was taken in the same temporal window in which $\alpha$ was also determined. This result strongly supports the notion that all trajectories were ergodic. In particular, our data separates well from the predictions of a non-truncated CTRW model that yields a lower bound $E(\alpha \leq 0.9) \geq 0.1{ }^{17}$ However, for the truncated CTRW model ( $c f$. ESI $\dagger$ ) we also obtained $E \approx 0.03$ on the experimentally relevant time scale. We attribute this effect to the truncation of $p(\tau)$ which narrows the distribution of apparent diffusion constants in $\left\langle r(t)^{2}\right\rangle_{T}{ }^{17,18}$ Hence, based only on $E$, a clear-cut decision that our experimental data is inconsistent with a truncated CTRW model is not possible.

We next inspected the trajectories' shape to gain deeper insights into the underlying type of random walk. The asphericity provides a simple yet powerful parameter to quantify the shape of fractal objects like random walks. ${ }^{27}$ Diagonalizing the random walk's gyration tensor $T_{i j}(c f$. ESI $\dagger$ ) yields the principal axes of gyration and 
the corresponding eigenvalues, i.e. the squared principal radii of gyration, $R_{i}^{2}$. Restricting ourselves to two dimensions (the experimental trajectories are two-dimensional objects), the asphericity is defined as

$$
A=\left\langle\left(R_{1}^{2}-R_{2}^{2}\right)^{2}\right\rangle /\left\langle\left(R_{1}^{2}+R_{2}^{2}\right)^{2}\right\rangle .
$$

We note that $A$ involves an averaging over the ensemble of walks (indicated by $\langle\ldots\rangle$ ). The limiting cases $A=0$ and $A=1$ resemble a perfect sphere and a simple rod, respectively. For Brownian motion in two dimensions an exact value is available: ${ }^{27} A=4 / 7$. Hence, even an individual trajectory of a two-dimensional Brownian random walk differs drastically from a circular shape at each instant of time. The time-averaged orientation of the longest principal axis of gyration, however, is isotropic. Moreover, the isotropy of diffusion is also recovered in an ensemble of particles due to the uncorrelated random orientations of the gyration axes.

We have determined via simulations the values of $A$ for FBM, OD, and a truncated CTRW at varying anomaly values $\alpha$ (see ESI $\dagger$ for details). Our simulation results revealed that for $0.5 \leq \alpha \leq 1$, which is the experimentally relevant regime, the asphericity changes almost linearly with $\alpha$, i.e. $A=m_{1} \alpha+b_{1}$. For OD we found $m_{1}=0.120 \pm$ $0.006, b_{1}=0.458 \pm 0.004$ whereas for FBM we obtained $m_{1}=$ $0.638 \pm 0.009, b_{1}=-0.057 \pm 0.006$. For the truncated CTRW model we found $A \approx 4 / 7$ irrespective of $\alpha$ (cf. ESI $\dagger$ ). This result can be rationalized by bearing in mind that a CTRW trajectory at any instance of time looks similar to the path of normal Brownian motion.

To compare our experimental trajectories to these predictions, we assigned the previously determined anomaly $\alpha$ to each trajectory ( $c f$. Fig. 1b). Then, we calculated the accompanying asphericity: Since the anomaly reflects a scaling for short and intermediate times, a consistent estimate of the random walk's asphericity must relate to the same time scale. Therefore, each trajectory was cut into sequences of $N=$ 3000 time steps of length $\Delta t$, and the average over these sub-trajectories yielded the (mean) asphericity [eqn (3)] of the entire trajectory on the length and time scales during which anomalous diffusion was observed. As can be seen from Fig. 2a, the cloud of data points for sucrose solutions overlaps well with the anticipated result for normal Brownian motion, i.e. the mean of all 21 data points $(\langle\alpha\rangle=0.98$ and $\langle A\rangle=0.58)$ agrees quantitatively with the expectation $\alpha=1$ and $A=$ $4 / 7 \approx 0.57$. Hence, sucrose solutions indeed feature normal Brownian trajectories also from the geometric perspective. In dextran solutions, however, we obtained $\langle\alpha\rangle=0.82$ and $\langle A\rangle=0.46$ which is most consistent with the simulation results for the FBM model that predicts locally a more spherical shape of the trajectory due to the anti-persistence of the random walk.

Given that FBM is closely related to the viscoelasticity of nonNewtonian and crowded fluids, ${ }^{13,28}$ the emergence of subdiffusion may be traced back to transient restoring forces on short length and time scales. It is hence meaningful to translate the SPT trajectories into the fluid's complex shear modulus, ${ }^{28} G(\omega)=G^{\prime}(\omega)+i G^{\prime \prime}(\omega)$. Here, the real (imaginary) part of $G(\omega)$ represents the elastic (viscous) modulus of the fluid. Employing a semi-analytical approach, we have fitted the time-averaged MSD of each trajectory by an empirical expression $w(t)=a_{0} t^{\alpha}+a_{1} t$ to capture the transient anomaly and the asymptotic normal diffusion. The resulting fit parameters were then used to determine the complex shear modulus as described earlier. ${ }^{13}$ From the ensemble of complex shear moduli for each fluid, we have determined the minimum and maximum values of $G^{\prime}$ and $G^{\prime \prime}$. As

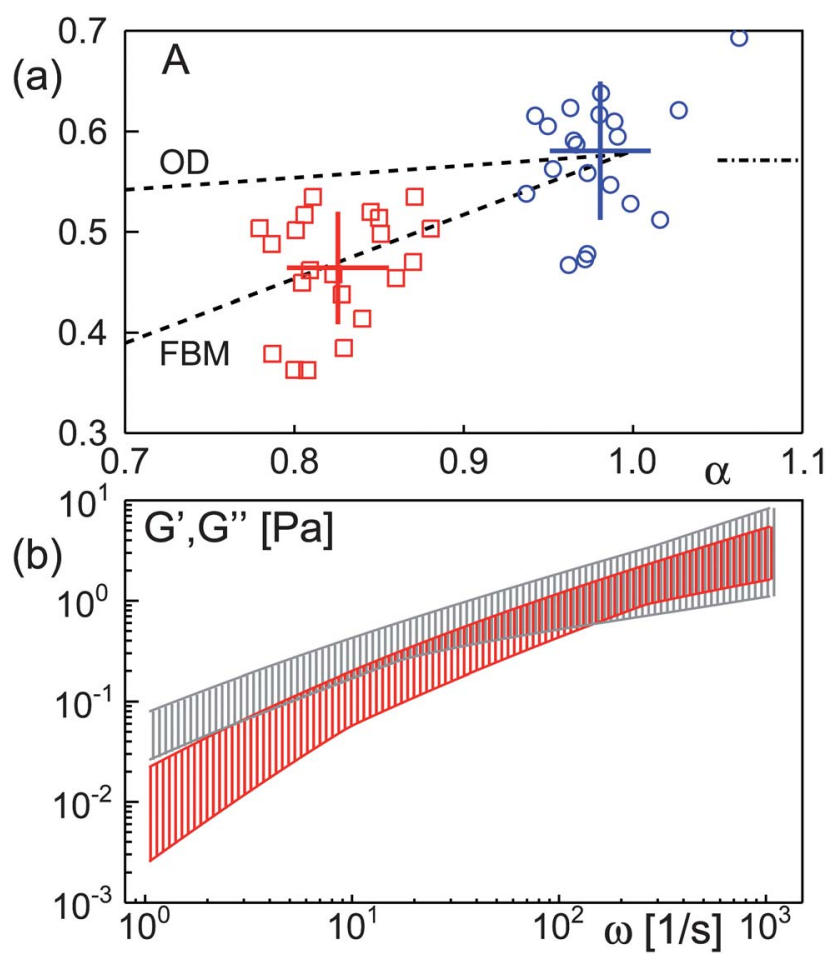

Fig. 2 (a) Asphericity $A$ as a function of the anomaly $\alpha$ (data for sucrose and dextran shown as blue circles and red squares, respectively). Mean values ( \pm standard deviation) are indicated by cross hairs. Dashed lines indicate simulation results for OD and FBM. Data for sucrose solutions are in very good agreement with the asymptotic value $A=4 / 7$ for $\alpha=1$ (dash-dotted mark), whereas data for dextran compare favorably to the predictions of FBM. (b) Elastic (red) and viscous (grey) moduli, $G^{\prime}$ and $G^{\prime \prime}$, as obtained from the ensemble of trajectories in a crowded dextran solution. Shown are the minimum and maximum values for $G^{\prime}$ and $G^{\prime \prime}$ at each frequency $\omega$, i.e. all trajectories lie within the indicated bands. For low frequencies the fluid is almost completely viscous whereas for $\omega>$ $100 \mathrm{~s}^{-1}$, a clear viscoelastic behavior emerges.

expected, sucrose showed a vanishing elastic contribution whereas the crowded dextran solution showed a significant viscoelasticity for large frequencies (Fig. 2b). Since high frequencies are related to small times, this viscoelastic behavior is intimately linked to the transient subdiffusion observed for small and intermediate times. A similar viscoelastic behavior (related to subdiffusion) has been observed for the cytoplasm and nucleoplasm of living cells. ${ }^{13,29}$

In conclusion, we have shown with an advanced SPT approach that a purely viscous sucrose solution features normal Brownian motion of tracer particles with an asphericity of the random walk that agrees very well with analytical predictions. In contrast, diffusion in a crowded dextran solution was anomalous ('subdiffusion'). Trajectories showed no signs of ergodicity breaking and their asphericity was in quantitative agreement with predictions of the FBM model. In contrast, obstructed diffusion (i.e., a standard random site percolation model) and CTRW were incompatible with the experimental data. This result is corroborated by the associated complex shear modulus: A strong viscoelastic behavior of the crowded dextran solution was seen at high frequencies as expected due to the relation of FBM with viscoelastic media.

It is tempting to speculate about the reasons and consequences of our finding in the context of living matter. Since the degree of 
cytoplasmic crowding appears to be conserved, ${ }^{29}$ cells might have adapted to highly crowded conditions and aim at maintaining this state ( $c f$. also discussion in ref. 2 and 29). Indeed, a potential benefit of FBM-like subdiffusion in cells is the increased return probability to a position in three-dimensional space. In particular, FBM with $\alpha<2 /$ 3 yields a bulk-filling random walk that can massively increase the capture probability to a target as compared to normal diffusion. ${ }^{30}$ Moreover, an enhanced rebinding due to FBM most likely is the explanation for the recently observed phosphorylation enhancement of MAPK under crowded conditions. ${ }^{3,4}$ As an enhanced recurrence is a generic feature of FBM-like subdiffusion, we expect that the behavior of a multitude of biochemical pathways in cells will have to be revisited and interpreted in light of our findings.

\section{Acknowledgements}

DE and JK gratefully acknowledge financial support by Research Unit FOR608. MH was partly financed by the German-Israeli Project Cooperation GA309/10. We would like to thank Stefan Hain for technical support.

\section{References}

$1 \mathrm{~J}$. Ellis and A. Minton, Nature, 2003, 425, 27-28.

2 H. Zhou, G. Rivas and A. Minton, Annu. Rev. Biophys., 2008, 37, 375-397.

3 K. Aoki, M. Yamada, K. Kunida, S. Yasuda and M. Matsuda, Proc. Natl. Acad. Sci. U. S. A., 2011, 108, 12675-12680.

4 M. Hellmann, D. Heermann and M. Weiss, Europhys. Lett., 2012, 97, 58004.

5 J. Dix and A. Verkman, Annu. Rev. Biophys., 2008, 37, 247-263.

6 M. Weiss, M. Elsner, F. Kartberg and T. Nilsson, Biophys. J., 2004, 87, 3518-24.
7 I. Tolic-Norrelykke, E. Munteanu, G. Thon, L. Oddershede and K. Berg-Sorensen, Phys. Rev. Lett., 2004, 93, 078102.

8 I. Golding and E. Cox, Phys. Rev. Lett., 2006, 96, 098102.

9 S. Weber, A. Spakowitz and J. Theriot, Phys. Rev. Lett., 2010, 104, 238102.

10 V. Tejedor, O. Bénichou, R. Voituriez, R. Jungmann, F. Simmel, C. Selhuber-Unkel, L. Oddershede and R. Metzler, Biohys. J., 2010, 98, 1364-1372.

11 I. Wong, M. Gardel, D. Reichman, E. Weeks, M. Valentine, A. Bausch and D. Weitz, Phys. Rev. Lett., 2004, 92, 178101.

12 D. Banks and C. Fradin, Biophys. J., 2005, 89, 2960-2971.

13 G. Guigas, C. Kalla and M. Weiss, Biophys. J., 2007, 93, 316-23.

14 W. Pan, L. Filobelo, N. Pham, O. Galkin, V. Uzunova and P. Vekilov, Phys. Rev. Lett., 2009, 102, 058101.

15 J. Szymanski and M. Weiss, Phys. Rev. Lett., 2009, 103, 038102.

16 M. Saxton, Biophys. J., 1994, 66, 394-401.

17 Y. He, S. Burov, R. Metzler and E. Barkai, Phys. Rev. Lett., 2008, 101, 058101 .

18 A. Lubelski, I. Sokolov and J. Klafter, Phys. Rev. Lett., 2008, 100, 250602.

19 M. Magdziarz, A. Weron, K. Burnecki and J. Klafter, Phys. Rev. Lett., 2009, 103, 180602.

20 J.-H. Jeon, V. Tejedor, S. Burov, E. Barkai, C. Selhuber-Unkel, K. Berg-Sorensen, L. Oddershede and R. Metzler, Phys. Rev. Lett., 2011, 106, 048103.

21 B. Hicks and K. Angelides, J. Membr. Biol., 1995, 144, 231-244.

22 T. Schmidt, G. Schutz, W. Baumgartner, H. Gruber and H. Schindler, Proc. Natl. Acad. Sci. U. S. A., 1996, 93, 2926-2929.

23 J. Enderlein, Appl. Phys. B: Lasers Opt., 2000, 71, 773-777.

24 K. Kis-Petikova and E. Gratton, Microsc. Res. Tech., 2004, 63, 34.

25 A. Berglund and H. Mabuchi, Appl. Phys. B: Lasers Opt., 2004, 78, 653.

26 Y. Katayama, O. Burkacky, M. Meyer, C. Brauchle, E. Gratton and D. Lamb, ChemPhysChem, 2009, 10, 2458-2464.

27 J. Rudnick and G. Gaspari, Science, 1987, 237, 384-9.

28 T. Mason and D. Weitz, Phys. Rev. Lett., 1995, 74, 1250-1253.

29 G. Guigas, C. Kalla and M. Weiss, FEBS Lett., 2007, 581, 5094-98.

30 G. Guigas and M. Weiss, Biophys. J., 2008, 94, 90-94. 